\title{
Ambiguous-Cue Interpretation is Biased Under Stress- and Depression-Like States in Rats
}

\author{
Thomas Enkel', Donya Gholizadeh', Oliver von Bohlen und Halbach ${ }^{2}$, Carles Sanchis-Segura $^{3}$, Rene \\ Hurlemann $^{4}$, Rainer Spanagel ${ }^{5}$, Peter Gass' and Barbara Vollmayr*,' \\ 'Department of Psychiatry and Psychotherapy, Central Institute of Mental Health, University of Heidelberg, Mannheim, Germany; ${ }^{2}$ nnstitute of \\ Anatomy \& Cell Biology, Ernst Moritz Arndt University of Greifswald, Greifswald, Germany; ${ }^{3}$ Area de Psicobiología, Universitat Jaume I, Campus \\ de Riu Sec, Castelló, Spain; ${ }^{4}$ Department of Psychiatry, University of Bonn, Bonn, Germany; ${ }^{5}$ Department of Psychopharmacology, Central \\ Institute of Mental Health, University of Heidelberg, Mannheim, Germany
}

Negative cognitive bias - the tendency to interpret ambiguous situations pessimistically — is a central feature of stress-related disorders such as depression. The underlying neurobiology of this bias, however, remains unclear, not least because of a lack of translational tools. We established a new ambiguous-cue interpretation paradigm and, with respect to the etiology of depression, evaluated if environmental and genetic factors contribute to a negative bias. Rats were trained to press a lever to receive a food reward contingent to one tone and to press another lever in response to a different tone to avoid punishment by electric foot-shock. In the ambiguous-cue test, the leverpress responses to tones with frequencies intermediate to the trained tones were taken as indicators for the rats' expectation of a positive or negative event. A negative response bias because of decreased positive and increased negative responding was found in congenitally helpless rats, a genetic animal model of depression. Moreover, treatment with a combined noradrenergic-glucocorticoid challenge, mimicking stress-related changes in endogenous neuromodulation, biased rats away from positive responding. This response shift was accompanied by neuronal activation in dentate gyrus and amygdala. Thus, environmental and genetic risk factors for depression induce a response bias, which resembles the pessimistic bias of patients suffering from depression. The behavioral paradigm described constitutes a useful tool to study the neuronal basis of decision making under ambiguous conditions and may promote innovative pharmaco- and psychotherapy for depression.

Neuropsychopharmacology (2010) 35, I008-1015; doi:I0.1038/npp.2009.204; published online 30 December 2009

Keywords: ambiguity; decision-making; depression; stress; rat

\section{INTRODUCTION}

Situations requiring decision making under uncertainty reveal the close interplay between cognitive and emotional processes. This becomes particularly evident in depression, a stress related and often life-threatening disorder affecting up to $20 \%$ of the population worldwide (Krishnan and Nestler, 2008). A major characteristic of this disorder is a negatively distorted interpretation of ambiguous information (Lawson et al, 2002; Chan et al, 2008; Dearing and Gotlib, 2009). The cognitive theory of depression emphasizes this negative cognitive bias toward pessimistic judgments as a key factor leading to the pathological condition (Beck, 2008). Understanding of the neurobiological mechanisms of this bias could thus provide a new

*Correspondence: Dr Barbara Vollmayr, Department of Psychiatry and Psychotherapy, University of Heidelberg, Central Institute of Mental Health, Mannheim, Baden-Wurttemberg 68159, Germany, Tel: + 496211703 2351, Fax: + 004962117032005 ,

E-mail: barbara.vollmayr@zi-mannheim.de

Received 27 August 2009; revised 21 October 2009; accepted 6 November 2009 approach for therapeutic intervention. However, although neurophysiological work in monkeys and functional neuroimaging in humans implicate amygdala, cingulate cortex, and several prefrontal regions in decision making under ambiguity and risk (Ernst and Paulus, 2005; Bach et al, 2009; Sugrue et al, 2005; Opris and Bruce, 2005; Chaudhry et al, 2009) the neurobiological mechanisms underlying the negative cognitive bias are yet poorly understood. Measuring corresponding patterns of behavior with similar methods could bridge the gap between the psychological phenomenon in humans and its underlying biological processes that can be fully examined only in animal models.

In a landmark study, Harding et al could show the existence of a cognitive bias in rats using a go/no-go task of ambiguous-cue interpretation (Harding et al, 2004). However, such a paradigm does not allow distinguishing whether a response bias originates from reduced positive and/or increased negative responding. Moreover, a 'no-go' as a response indicator cannot be distinguished from a response omission. We, therefore, addressed this problem in our study by developing a new go/go-task, in which we 
trained rats to press a lever to receive a food reward contingent to one tone and to press another lever in response to a different tone to avoid contingent punishment by electric foot-shock. Subsequently, rats were tested for their responses to ambiguous probe tones of intermediate frequencies and their expectations of a positive or negative event signalled by these tones were inferred from their lever-press responses (Figure 1).

An animal model derived from the cognitive theory of depression is 'learned helplessness', in which the experience of uncontrollable stress leads to a helpless state with depression-like symptoms (Overmier and Seligman, 1967). By selectively breeding helpless and non-helpless animals, two different rat lines could be established: rats that congenitally show helpless behavior (cLH rats) and rats that show relative resistance to learned helplessness (cNLH rats). These strains differ in neurochemical and behavioral parameters that are related to depression (Henn and Vollmayr, 2005). In a first experiment, we analyzed whether the helpless phenotype of the cLH rats would manifest in the described task as a more negative pattern of tone interpretation.

Apart from a genetic predisposition, environmental factors contribute to at least $50 \%$ to the etiology of depression (Krishnan and Nestler, 2008). Stress constitutes a major risk factor for depression and stress-induced alterations in endogenous neuromodulator functioning have been implicated in the depressive pathophysiology (De Kloet et al, 2005). Interestingly, recent findings suggest an amygdala activation bias to negative stimuli, using noradrenergic-glucocorticoid challenges to pharmacologically mimic neurobiological stress conditions (Kukolja et al, 2008), which underscores the potential of stress to negatively bias neural function. To validate whether such alterations effect on our behavioral model, we carried out a second experiment to assess ambiguous-cue interpretation in rats co-treated with the noradrenaline reuptake inhibitor Reboxetine (Rbx) and corticosterone (Cort), the rodent analogon to cortisol. In addition, to control if treatmentinduced changes in cue interpretation were related to alterations in neuronal activity, we quantified c-Fos immunoreactivity in several nuclei of the amygdala and in the hippocampal dentate gyrus.

\section{MATERIALS AND METHODS}

\section{Animals}

For ambiguous-cue interpretation in congenitally helpless and non-helpless rats ('cLH $v s$ cNLH' experiment), 16 eightweek-old male cLH $(n=8)$ and $\mathrm{cNLH}(n=8)$ rats from different litters of the sixty-fifth generation of the colonies bred in Mannheim were used. Both, $\mathrm{cLH}$ and $\mathrm{cNLH}$ strains were bred from Sprague-Dawley rats. Approximately $90 \%$ of the cLH rats typically express the helpless phenotype. Routinely, learned helplessness is assessed by testing animals for deficits in foot-shock escape behavior. As we wanted to have shock-naïve animals in this study, the phenotype was controlled by showing anhedonia (as reduced SCM intake, see below). Learned helplessness (non-helplessness, respectively) was additionally confirmed in litter mates of the experimental animals. The origin and selective breeding of both strains has been described earlier (Henn and Vollmayr, 2005; Vollmayr and Henn, 2001). In the second Experiment ('Rbx + Cort'), we used 36 male wildtype Sprague-Dawley rats (Elevage Janvier, France) to assess the effects of Rbx and Cort co-treatment on ambiguous-cue interpretation $(n=16)$ and reward intake $(n=20)$.

All rats were group housed in macrolon cages (type 4) and kept under controlled conditions (room temperature: $22{ }^{\circ} \mathrm{C}$; lights on from 0800 to 2000 hours); food and water were available ad libitum. The experimental protocols used in this study were in line with national and international ethical guidelines, conducted in compliance with the German Animal Welfare Act and approved by the Regierungspräsidium Karlsruhe, including approval by an animal ethics committee.

\section{Drugs}

Corticosterone-HBC complex (Sigma-Aldrich, Schnelldorf, Germany) was dissolved in saline and administered via
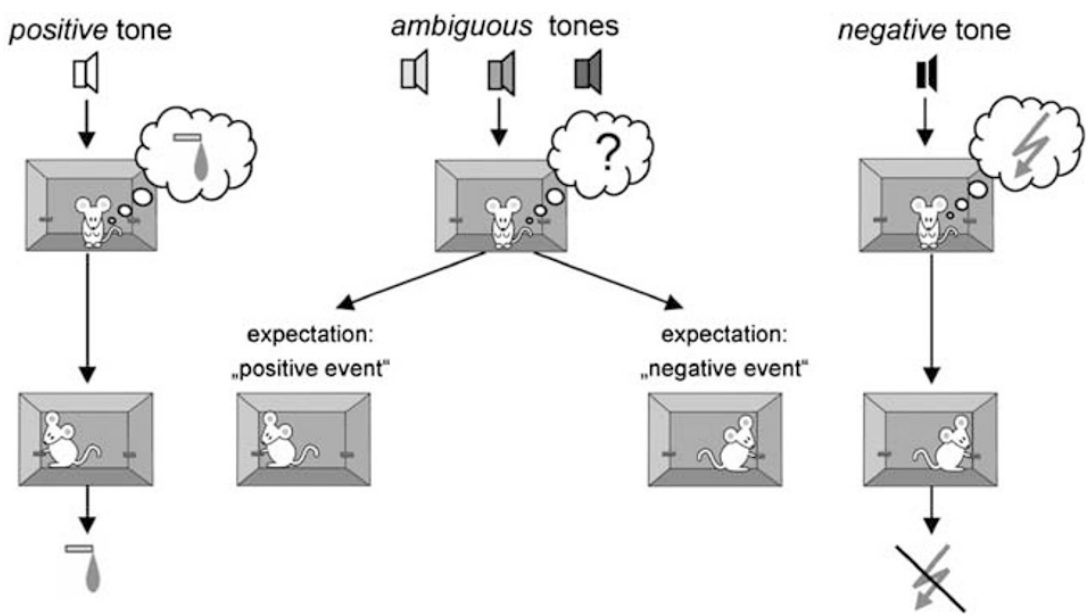

Figure I Behavioral protocol. A positive tone ( 2 or $9 \mathrm{kHz}$ tone; counterbalanced with negative tone) signalled the opportunity to gain a reward (sweetened condensed milk) by pressing the left lever. A negative tone preceded the occurrence of an electric foot-shock, which could be prevented by pressing the right lever. After discrimination training rats were tested for their responses to ambiguous tones with intermediate frequencies $(3,5$, and $7 \mathrm{kHz}$ ). The rats' expectations of a positive or a negative event signalled by these tones were inferred from their lever responses. 
intraperitoneal injection $30 \mathrm{~min}$ before behavioral testing $(0.5 \mathrm{mg} / \mathrm{kg}$ in $1 \mathrm{ml} / \mathrm{kg})$. Rbx $(15 \mathrm{mg} / \mathrm{kg}$ in $2 \mathrm{ml} / \mathrm{kg}$; Merz Pharmaceuticals, Frankfurt, Germany) was dissolved in water and administered via gastric gavage $60 \mathrm{~min}$ before behavioral testing. To minimize handling stress, rats were well trained to this type of substance application. Sweetened condensed milk (SCM, Milchmaedchen, Nestle, Germany) was diluted in water (mixing ratio $1: 3$ ) and used as rewarding reinforcer in all behavioral experiments.

\section{Limited Access Sweetened Condensed Milk Intake}

Non-deprived cLH and cNLH rats were separated in small cages (type 3) to which they had been habituated $24 \mathrm{~h}$ before. After $5 \mathrm{~min}$ of habituation, rats had $15 \mathrm{~min}$ of free access to the SCM solution. The amount of liquid consumed by each rat was determined by weighing the bottles and calculated in relation to the individual body weight. In rats receiving $\mathrm{Rbx}+$ Cort $(n=10)$ or saline $(n=10)$, SCM intake was tested in two sessions (under baseline and treatment conditions) to assess effects of drug treatment or handling stress (because of gastric gavage or i.p. injection) on the hedonic value of the SCM reward.

\section{Ambiguous-Cue Test}

Apparatus. The behavioral tasks were carried out in eight computer-controlled skinner boxes (MedAssociates, St Albans, Vermont, USA), each equipped with two retractable levers and liquid dispensers (set to deliver $\sim 100 \mu \mathrm{l} \mathrm{SCM}$ ) located on opposite walls, a grid floor through which scrambled electric shocks $(\sim 700 \mu \mathrm{A})$ could be delivered and a speaker. All behavioral protocols described below, including stimulus presentation and data acquisition, were programmed in MedState Notation code (MedAssociates).

Positive tone training. After handling rats were trained to press the lever attached to the left wall of the skinner box to receive $\mathrm{SCM}$ when a tone $(30 \mathrm{~s}, 2 \mathrm{kHz}$ at $75 \mathrm{~dB}$ sound pressure level (SPL) or $9 \mathrm{kHz}$ at $63 \mathrm{~dB}$ SPL (counterbalanced)) signalled reward availability. Owing to the association to food intake, this tone acquired positive valence and is referred to as the 'positive tone' with the associated lever termed the 'positive lever'. During presentation of this tone, pressing the left lever was continuously rewarded by delivery of $80 \mu \mathrm{l} \mathrm{SCM}$. After $30 \mathrm{~s}$ the tone was turned off and an intertrial interval (ITI) began during which lever pressing was not rewarded. The ITI was progressively increased from $30 \mathrm{~s}$ on the first day to $120 \mathrm{~s}$ on the last day. After five daily 30-min sessions, this schedule yielded focused responding during tone presentations. On the sixth day of training, in contrast to the preceding days, a lever press within the 30-s tone presentation was rewarded with SCM delivery but terminated the tone and started a 90-s ITI.

Negative tone training. Subsequently rats were trained to respond to another tone $(9 \mathrm{kHz}$ at $63 \mathrm{~dB} S P L$ or $2 \mathrm{kHz}$ at $75 \mathrm{~dB}$ SPL (counterbalanced)) in an active lever-press avoidance schedule. A presentation of this tone was paralleled by the occurrence of electric shocks $30 \mathrm{~s}$ after tone onset, unless the rat pressed the lever attached to the right wall of the skinner box, which prevented onset of the shock and terminated tone presentation ('avoidance response'). Pressing the lever after shock-onset terminated the shock and tone presentation ('escape response'). Owing to the association to an aversive stimulus, this tone acquired negative valence and is referred to as the 'negative tone', with the associated lever termed the 'negative lever'. Maximum duration of tone/shock-application was $90 \mathrm{~s}$ (ie, $60 \mathrm{~s}$ of shock) and trial presentations were separated by 90 -s ITIs. Daily training sessions consisted of 20 trials. During the first day of training the rats were assisted in escaping electric shocks by gently pushing them toward the lever. Rats had to accomplish at least $60 \%$ correct avoidance responses before proceeding with discrimination training.

Discrimination training. During discrimination training, 10 positive and negative tone trials were presented in a pseudo randomized order, separated by 90 -s ITIs. A trial was considered to be correct when a rat carried out the response associated with each tone (as learned in the earlier stages of training) within $30 \mathrm{~s}$ of stimulus presentation. Pressing the wrong lever (eg, pressing the right instead of the left lever in response to a positive tone presentation) was considered as a failure. No responding (omissions) or escape responses were also considered as failure trials. Training was continued until rats accomplished a stable baseline of correct discrimination responses on at least $70 \%$ of the trials.

Ambiguous-cue testing. Ambiguous-cue testing consisted of a discrimination task session as described above, with presentation of three additional probe tones with frequencies intermediate between the positive and negative tones: $3 \mathrm{kHz}$ at $72 \mathrm{~dB}$ SPL, $5 \mathrm{kHz}$ at $68 \mathrm{~dB}$ SPL, and $7 \mathrm{kHz}$ at $65 \mathrm{~dB}$ SPL. Frequencies were chosen to be roughly equidistant on a logarithmic scale. Owing to the fact that the hearing threshold varies across frequencies, loudness of the tones was roughly adapted according to the audiogram of Sprague-Dawley rats (Kelly and Masterton, 1977). The probe tones were named in relation to the trained tones as 'near-positive', 'medial', and 'near-negative'. Within a test session, two probe tones of each frequency were intermixed with nine positive and negative tone presentations. Any lever press to a probe tone within 30-s terminated tone presentation but had no consequences. If a rat did not respond within $30 \mathrm{~s}$, the tone was terminated and a 'response omission' was scored. The computer recorded the side of lever pressing to each tone, latency to press the lever and the number of intertrial lever presses.

In experiment ' $\mathrm{CLH} v s \mathrm{CNLH}$ ', the rats were tested for ambiguous-cue interpretation on 6 consecutive days. In experiment ' $\mathrm{Rbx}+$ Cort', each rat was treated with both, the combination $\mathrm{Rbx}+$ Cort or saline in a cross-over design, thereby serving as its own control. For 3 days of ambiguouscue interpretation, one-half of the rats received $\mathrm{Rbx}+\mathrm{Cort}$, while the other half received sham treatment with saline. Four weeks later, this treatment scheme was reversed and ambiguous-cue interpretation was again tested in three consecutive sessions. To ensure adequate discrimination performance, rats received 3 days of discrimination training before to the second ambiguous-cue testing session (Figure 2). 


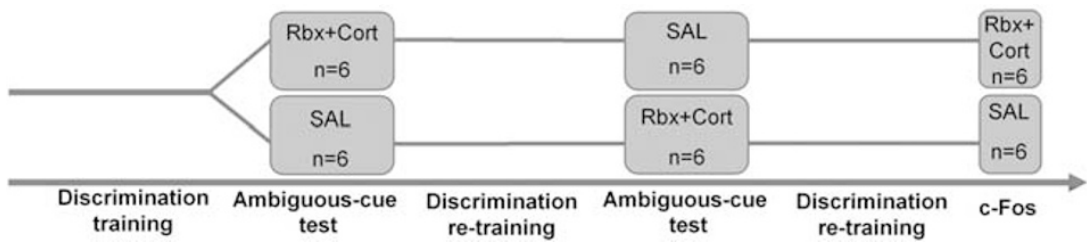

Figure 2 Timeline depicting the design of the Reboxetine + Corticosterone experiment. All animals $(n=12)$ were trained in the discrimination task. For ambiguous-cue testing, they were split into two groups; one receiving Rbx + Cort treatment, the other one receiving sham treatment with saline. Four weeks later, rats were retrained in the discrimination task to ensure adequate discrimination performance and were then tested again in the ambiguous-cue test under a reversed treatment schedule. Finally, one additional ambiguous-cue test was performed to stimulate brain circuits for c-Fos analysis.

\section{c-Fos Immunoreactivity}

The expression of the immediate early gene c-Fos was quantified in the same rats that were used for behavioral testing. An additional ambiguous-cue test was run as a behavioral challenge to stimulate participating brain circuits after completion of behavioral testing (data not included in the behavioral analysis). Half of the rats were treated with $\mathrm{Rbx}+$ Cort, the other half received sham treatment with saline. Ninety minutes after starting the test, animals were transcardially perfused with a paraformaldehyde-PBS solution and brains were removed. Coronal brain sections $(50 \mu \mathrm{m})$ were cut and processed for c-Fos immunoreactivity. A detailed description of the procedure can be found in the Supplementary Methods.

\section{Data Analysis}

Rats that showed declining discrimination performance below 50\% during ambiguous-cue testing were excluded from analysis to avoid confounding effects on cue interpretation because of poor tone discrimination. Responding to each tone (positive, near-pos, medial, near-neg, or negative) was analyzed separately by calculating the proportions of tones responded to by pressing the positive or negative lever or responded to with omissions. A multivariate analysis of variance (MANOVA) using Wilk's Lambda test was applied to analyze effects of the factors 'strain' (in 'cLH vs cNLH experiment) or 'treatment status' (in the $\mathrm{Rbx}+$ Cort experiment) on overall tone interpretation, followed by separate unpaired ('cLH vs cNLH') or paired $(\mathrm{Rbx}+\mathrm{Cort}) t$-tests for each tone.

To calculate individual relative response preferences, negative responding was subtracted from positive responding, resulting in positive values (between 0 and 1) for a positive response preference and negative values (between 0 and -1 ) for a negative response preference. An overall response preference was then determined by an area under curve analysis for each rat and comparing groups using a paired ( $\mathrm{Rbx}+\mathrm{Cort})$ or unpaired ('cLH vs cNLH') $t$-test; $t$-tests were also used to analyze group differences in the c-Fos immunoreactivity experiment.

Data on SCM consumption were analyzed using an unpaired $t$-test ('cLH vs cNLH') or a repeated-measure ANOVA ( $\mathrm{Rbx}+$ Cort experiment) with factors treatment (Rbx + Cort/saline) and session (baseline/treatment). A $p$-value $<0.05$ was considered to indicate significant effects. All statistical procedures were performed using a standard software application (SPSS 15.0 for Windows, SPSS GmbH Software, Germany).

\section{RESULTS}

\section{cLH vs cNLH: SCM Consumption Test}

Sweetened condensed milk intake (in \% bodyweight) was significantly lower in cLH rats than in cNLH rats (cNLH: $2.32 \pm 0.08 \%$ SEM; cLH: $1.98 \pm 0.08 \%$ SEM; $t$-Test: $p<0.05)$. Bodyweight was not different between the two groups (cNLH: $615 \pm 19$ g SEM; cLH: $586 \pm 14$ g SEM).

\section{cLH vs cNLH: Task Acquisition}

Eleven rats (cNLH:6; cLH:5) reached the criterion of at least $70 \%$ correct discrimination trials over 3 consecutive days, with a similar average discrimination performance in both groups of rats (cNLH: $76 \pm 6 \%$ SEM, cLH: $81 \pm 3 \%$ SEM). Despite their differences in voluntary SCM consumption, $\mathrm{cLH}$ and $\mathrm{cNLH}$ did not differ in the number of SCM rewarded responses during discrimination training. Behavioral data from all training stages can be found in Supplementary Table 1.

\section{cLH vs cNLH: Ambiguous-Cue Test}

A MANOVA on ambiguous tones revealed a significant effect of the factor 'strain' on positive (Wilks- $\Lambda: \mathrm{F}_{3,6}=6.8$, $p<0.05$; Figure 3a) and negative lever-press responding (Wilks- $\Lambda: \mathrm{F}_{3,6}=4.85, p<0.05$; Figure $3 \mathrm{~b}$ ). Follow-up testing indicated that $\mathrm{cLH}$ rats made less positive lever-press responses to the medial $(p<0.05)$ and near-negative $(p<0.05)$ tones, whereas making more negative lever presses to the medial tone than cNLH rats $(p<0.05)$. This response shift resulted in a significantly more negative overall lever-press preference in rats of the $\mathrm{cLH}$ strain compared with cNLH rats $(p<0.01$; Figure $3 \mathrm{~d})$. No significant differences were found for the number of omissions (Wilks- $\Lambda: \mathrm{F}_{3,6}=0.304, p>0.8$; Figure 3c) and the mean number of lever presses per ITI were similar in the two groups (cNLH: $0.7 \pm 0.1$ SEM, cLH: $0.8 \pm 0.1$ SEM).

\section{Rbx + Cort: Task Acquisition}

Twelve rats reached the criterion of $70 \%$ correct discrimination trials and showed stable performance over 3 consecutive days of training. Discrimination performances before the first $(79 \pm 2.2 \%$ SEM $)$ and second $(81 \pm 3.4 \%$ SEM $)$ test of ambiguous-cue interpretation were similar, therefore justifying the use of a cross-over design. Behavioral data from all training stages can be found in Supplementary Table 2 . 

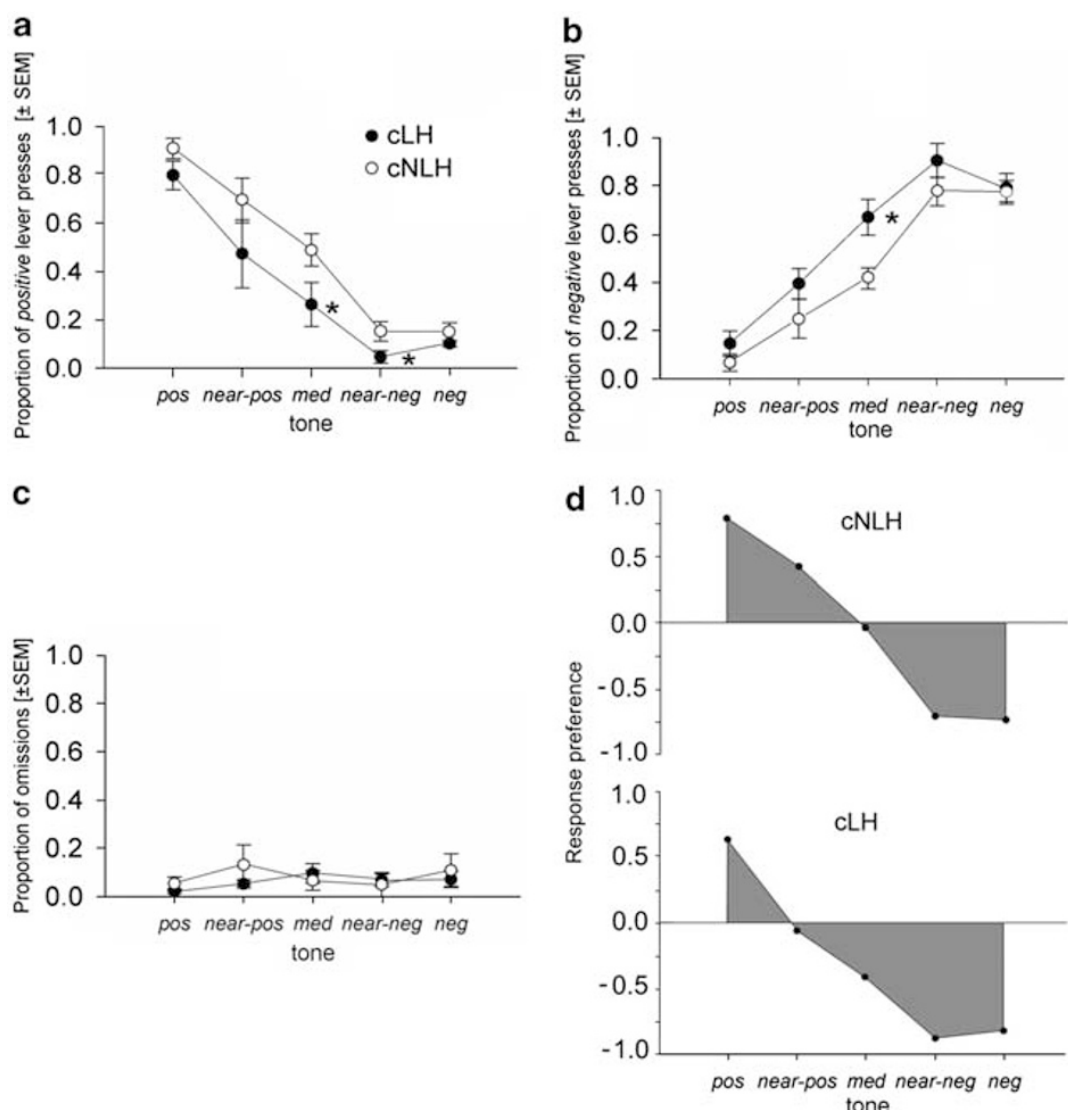

Figure $3(\mathrm{a}-\mathrm{c})$ Congenitally helpless rats $(\mathrm{cLH} ; n=4)$ showed decreased positive and increased negative responding to intermediate probe tones $(* p<0.05)$ when compared with non-helpless rats $(\mathrm{cNLH} ; n=6)$. There was no difference in the number of omissions. Data are proportions of positive- or negative-lever presses or omissions made to trained (pos and neg) and probe tones (near-pos, med, and near-neg). (d) These response shifts resulted in a more negative overall response preference $(p<0.0 \mathrm{I})$; a value of I or $-\mathrm{I}$ indicates a maximal preference for the positive or negative lever, respectively.

\section{Rbx + Cort: SCM Consumption Test}

Statistical testing revealed no significant effects of the factors treatment $\left(\mathrm{F}_{1,18}=0.06 ; p=0.808\right)$ or session $\left(\mathrm{F}_{1,18}=2.49 ; p=0.132\right)$ and there was no interaction of these factors $\left(\mathrm{F}_{1,18}=0.09 ; p=0.767\right.$; see Supplementary Table 3).

\section{Rbx + Cort: Ambiguous-Cue Test}

A repeated-measures MANOVA revealed a significant effect of the treatment status on positive lever pressing (Wilks$\Lambda: \mathrm{F}_{5,7}=3.98, p<0.05$; Figure $4 \mathrm{a}$ ) and on omissions (Wilks$\Lambda: \mathrm{F}_{5,7}=10.23, p<0.01$;Figure $\left.4 \mathrm{c}\right)$. Post hoc analysis for positive lever pressing indicated significantly less positive responding under $\mathrm{Rbx}+$ Cort treatment for the positive $(p<0.05)$, near-positive $(p<0.01)$, medial $(p<0.05)$, nearnegative $(p<0.05)$, and negative $(p<0.05)$ tones. Post hoc analysis for omissions indicated an $\mathrm{Rbx}+$ Cort induced increase in the number of omissions for the positive $(p<0.05)$, near-positive $(p<0.01)$, and medial $(p<0.05)$ tones. This response shift resulted in a significantly more negative overall lever-press preference $(p<0.01$; Figure $4 \mathrm{~d})$. The increase in the number of negative responses was not significant (Wilks- $\Lambda: \mathrm{F}_{5,7}=0.39, p>0.162$; Figure $4 \mathrm{~b}$ ). The number of intertrial lever presses and the response latencies were similar under both treatments (see Supplementary Table 4).

\section{Rbx + Cort: c-Fos Immunoreactivity Under Rbx + Cort Treatment}

Quantification of c-Fos immunoreactivity resulted in higher densities of c-Fos positive cells in the lateral amygdala, the basolateral amygdala, and the dentate gyrus of Rbx + Corttreated rats $(p<0.01, p<0.001$, and $p<0.01$, respectively; Figures $5 a$ and $b$ ). No significant differences were found for the medial and central amygdala.

\section{DISCUSSION}

Using an ambiguous-cue interpretation task, we show the existence of a negative response bias in rats congenitally showing a depressive-like trait. Furthermore, an acute pharmacologically induced increase in synaptic levels of noradrenalin and corticosterone resulted in a response shift away from positive responding, which was accompanied by changes in neuronal activity in the amygdala and the dentate gyrus.

The typical response pattern obtained in this task shows high response rates to the trained tones with usually a somewhat higher responding to the positive tone. For the ambiguous tones the response pattern reflects their level of ambiguity, because for the slightly ambiguous near-positive or near-negative tones the response rates clearly align to the trained tones and the response rate to the highly ambiguous medial tone being intermediate between them. Thus, tone 

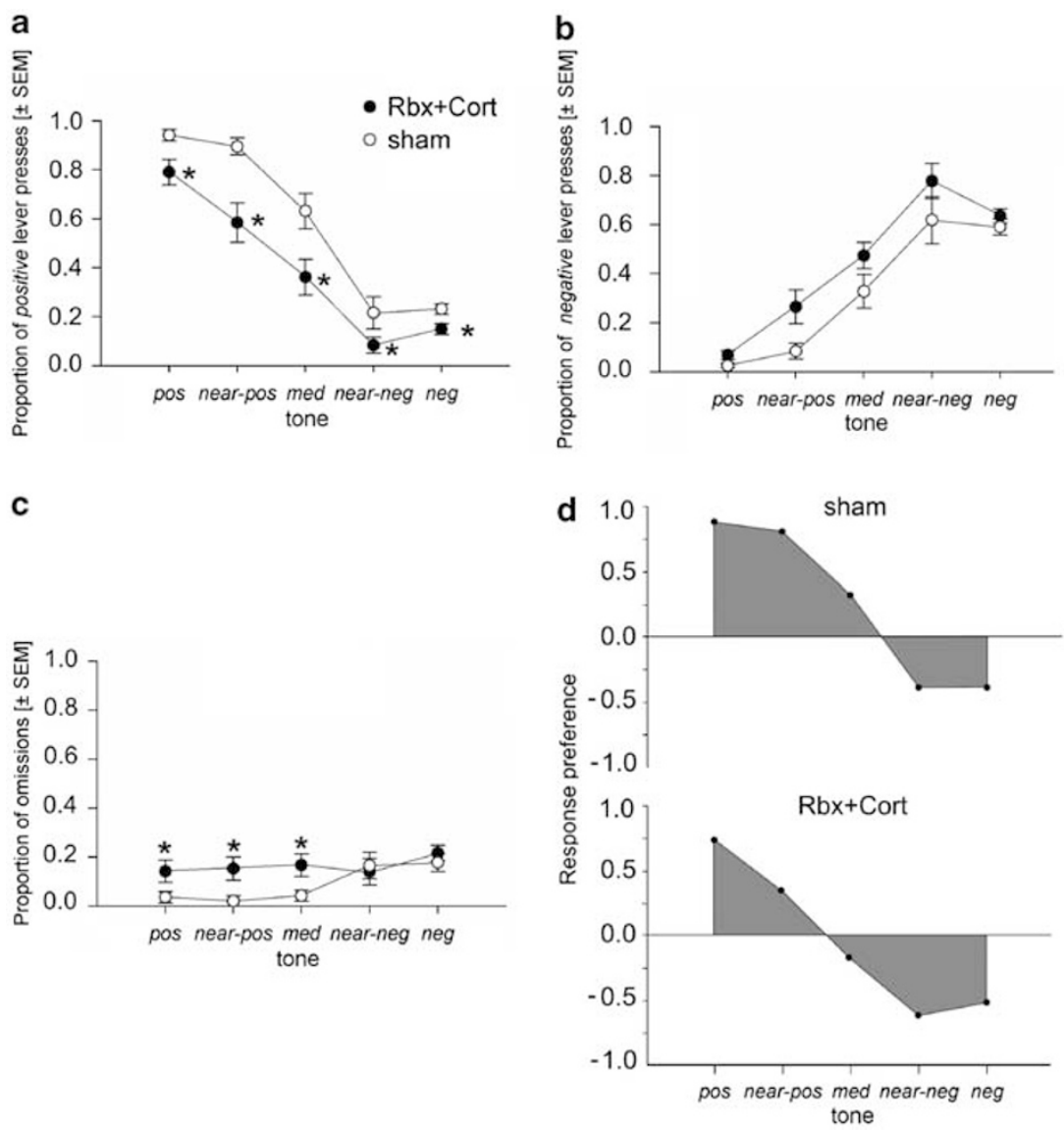

Figure $4(a-c)$ Under Rbx + Cort treatment, rats $(n=12)$ showed decreased positive responding and an increase of omissions $(* p<0.05)$, while negative responding was not significantly changed. Data are proportions of positive- or negative-lever presses or omissions made to trained (pos and neg) and probe tones (near-pos, med, and near-neg). (d) These response shifts resulted in a more negative overall response preference $(p<0.0 \mathrm{I})$; a value of $\mathrm{I}$ or $-\mathrm{I}$ indicates a maximal preference for the positive or negative lever, respectively.

interpretation was related to the learned 'valence-lever' associations and rats could dynamically adjust their responding to different levels of certainty. Interestingly, the response pattern observed for positive responding is similar to the one that Harding et al obtained with their go/ no-go task (Harding et al, 2004).

The effect of a depressive-like trait on ambiguous-cue interpretation was assessed in cLH rats, a genetic model of depression. Compared with cNLH rats, we found decreased positive-lever responses and increased negative-lever responses to ambiguous tones (particularly the medial tone) in cLH rats, resulting in a negative overall response pattern. These results were not because of global activity deficits in cLH rats as there were no between-group differences in response omissions or intertrial activities. The presence of the depressive-like phenotype was verified by showing decreased voluntary SCM consumption, a measure for the hedonic aspects of rewards (Slattery et al, 2007; Ellenbroek and Cools, 2000). However, cLH and cNLH rats neither differed in the amount of operant responses for SCM (presumably because operant demands were low) nor in their ability to discriminate the contingencies predicted by the positive and negative tones, ruling out significant motivational deficits during discrimination training. Thus, the shift in the response pattern to ambiguous tones observed in cLH rats arises from their responding to neutral stimuli as if they were predictors of aversive consequences, resembling the pessimistic bias of patients suffering from depression (Lawson et al, 2002; Chan et al, 2008). The finding of a negative response bias in cLH rats is consistent with results of Harding et al (2004), who report a negative response bias, which they could relate to decreased positive responding in normal rats after inducing a depressive-like state by chronic uncontrollable stress.

The fact that $\mathrm{cLH}$ rats show a response bias only to ambiguous information with unaltered processing of the emotional values of the trained tones implicates an involvement of neuronal circuits particularly related to decision making under ambiguity and risk, such as the anterior cingulate, prefrontal, and parietal cortices (Elliott et al, 1999; Opris and Bruce, 2005; Sugrue et al, 2005; Bach et al, 2009). It is noteworthy that cLH rats, such as depressed humans, show abnormal metabolism in some of these regions (Shumake et al, 2000).

An important observation is that helpless rats can learn avoidance responding despite their predisposition for deficient escape behavior. Avoidance learning was facilitated by the fact that during the first trials the rat's attention was explicitly directed toward the lever by the experimenter. Furthermore, training rats to press the positive lever for SCM preceded avoidance training and it has early been noted that learning about contingencies 
a

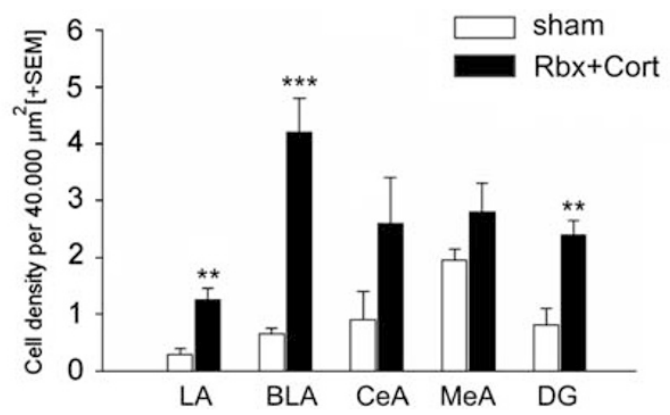

b
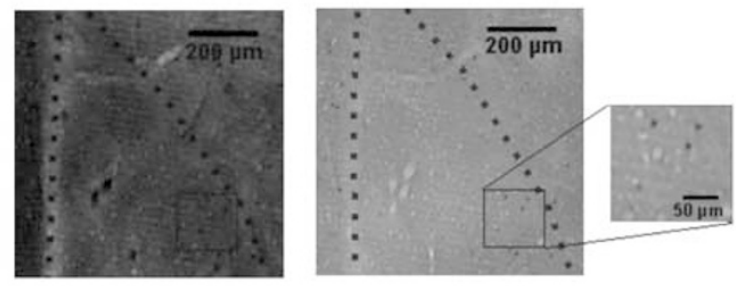

dentate gyrus

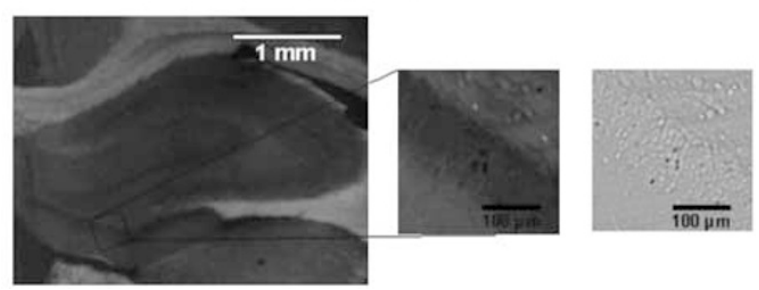

Figure 5 (a) Rbx + Cort treatment increased the number of c-Fos immunoreactive cells in the lateral $(L A)$ and basolateral $(B L A)$ nucleus of the amygdala and in the dentate gyrus (DG; $* * * 0.01$, **** $p<0.00$ I; CeA: central nucleus of the amygdala; MeA: medial nucleus of the amygdala); Rbx + Cort: $n=6$, sham: $n=6$. (b) Photomicrographs of the BLA and DG: $3^{\prime}$ 3'-diaminobenzidine-tetrahydrochloride (DAB)-induced fluorescence (in green) was used to define precisely the localization of the analyzed structures. After delineation of the structures, c-Fos immunoreactivity visualized by DAB (brownish non-fluorescent color) was used for counting. (See online version for color figure.)

between a self-performed action and an associated outcome is an immunization against helpless behavior (Seligman et al, 1975). Thus, our results implicate that although helpless rats learn to actively cope with aversive events, in ambiguous situations their behavior is still guided by the expectation of negative consequences.

Apart from genetic vulnerability, environmental factors such as stress-induced alterations in endogenous neuromodulator functioning are implicated in the depressive pathophysiology (De Kloet et al, 2005). Mimicking these alterations by a combined pharmacological potentiation of noradrenaline and cortisol has recently been shown to induce a negative bias in the human amygdala (Kukolja et al, 2008). In rats, the combined $\mathrm{Rbx}+$ Cort treatment decreased positive-lever responses paralleled by an increase in response omissions, but with only a slight, nonsignificant increase in negative-lever responding. Thus, other than in cLH rats, the more negative overall response pattern under $\mathrm{Rbx}+$ Cort treatment was mainly due to a response bias away from positive responding. Given that under sham treatment rats were more likely to respond to the medial tone with positive than negative-lever presses, our results might be interpreted as a decrease of a natural preference for positive responding. Interestingly, it has been shown that depressed humans have an impaired capacity to develop a response bias toward rewarding stimuli (Pizzagalli et al, 2008). Another important implication of these data are the necessity to distinguish between reduced positive and increased negative responding in ambiguouscue interpretation, because these might be two dissociable phenomena that can independently contribute to a response bias and may therefore be mediated by different neuronal processes.

It is noteworthy that decreased positive responding was not limited to probe tones but generalized to trained tones. However, as the results from the SCM consumption test most likely exclude drug- or handling-induced anhedonia as an explanation, we suggest this response bias reflects decreased positive associations with the tones. The pattern of omissions, showing significant increases only to the positive, near-positive, and medial tones, indirectly supports this view because such a negative shift would more likely decrease the motivation to respond to (potentially) positive, but not to negative cues. A general motivational deficit appears unlikely, because one would then expect lower responding also on the negative lever, which was not the case. Similar intertrial activities and response latencies far below the time limit of $30 \mathrm{~s}$ under sham as well as $\mathrm{Rbx}+$ Cort treatment, further rule out reduced activity or arousal levels. Finally, the treatment did also not affect response accuracy, leaving the learned associations between the tones and their corresponding lever-press responses intact.

Neuroanatomical analysis revealed a higher density of c-Fos immunoreactive cells in the lateral and basolateral amygdala and in the hippocampal dentate gyrus of $\mathrm{Rbx}+$ Cort treated rats, linking the observed bias to increased neuronal activity in these regions. The amygdala has been suggested to have a role in valence representations (Paton et al, 2006) and also in the processing of ambiguity (Rosen and Donley, 2006). Amygdaloid hyperactivity has been reported in subjects who are at high risk for anxiety and depression through environmental factors (Wolfensberger et al, 2008). Dentate gyrus hyperactivity has been implicated in overgeneralization of ambiguous aversive cues (Tsetsenis et al, 2007).

In conclusion, both a pharmacological challenge mimicking acute stress-like conditions and genetic risk factors effect on ambiguous cue interpretation in rats, inducing distinct forms of response biases. The task described is suitable to analyze the neuronal mechanisms of decision making under ambiguity and offers the opportunity to analyze the basis of a cognitive bias with respect to changes in positive and/or negative responsiveness. Being analog to human studies, this translational tool might promote innovative therapeutic treatment for depression.

\section{ACKNOWLEDGEMENTS}

We thank A Meyer-Lindenberg, B Krumm, and C Hiemke for valuable discussion, the unknown reviewers for helpful comments and $\mathrm{H}$ Schamber and $\mathrm{C}$ Dormann for technical support. BV, and $\mathrm{RH}$ were supported by the German Federal Ministry of Education and Research (BMBF) as a part of the research consortium 'The neurofunctional basis 
of emotion-cognition coupling'. In addition, $\mathrm{RH}$ was supported by a Starting Independent Researcher Grant provided by the Ministry of Innovation, Science, Research and Technology of the State of North Rhine-Westphalia (MIWFT). PG was supported by the Deutsche Forschungsgemeinschaft (SFB 636/B3). CS-S was supported by a contract of the Ramón y Cajal program from the Spanish Ministerio de Ciencia e Innovación.

\section{DISCLOSURE}

Dr Spanagel holds research and consultant contracts with Abbott, GSK, Organon, Solvay and XenoPort. All other authors declare no conflict of interest.

\section{REFERENCES}

Bach DR, Seymour B, Dolan RJ (2009). Neural activity associated with the passive prediction of ambiguity and risk for aversive events. J Neurosci 29: 1648-1656.

Beck AT (2008). The evolution of the cognitive model of depression and its neurobiological correlates. Am J Psychiatry 165: 969-977.

Chan SW, Harmer CJ, Goodwin GM, Norbury R (2008). Risk for depression is associated with neural biases in emotional categorisation. Neuropsychologia 46: 2896-2903.

Chaudhry AM, Parkinson JA, Hinton EC, Owen AM, Roberts AC (2009). Preference judgements involve a network of structures within frontal, cingulate and insula cortices. Eur J Neurosci 29: 1047-1055.

De Kloet ER, Joels M, Holsboer F (2005). Stress and the brain: from adaptation to disease. Nat Rev Neurosci 6: 463-475.

Dearing KF, Gotlib IH (2009). Interpretation of ambiguous information in girls at risk for depression. J Abnorm Child Psychol 37: 79-91.

Ellenbroek BA, Cools AR (2000). Animal models for the negative symptoms of schizophrenia. Behav Pharmacol 11: 223-233.

Elliott R, Rees G, Dolan RJ (1999). Ventromedial prefrontal cortex mediates guessing. Neuropsychologia 37: 403-411.

Ernst M, Paulus MP (2005). Neurobiology of decision making: a selective review from a neurocognitive and clinical perspective. Biol Psychiatry 58: 597-604.

Harding EJ, Paul ES, Mendl M (2004). Animal behaviour: cognitive bias and affective state. Nature 427: 312.

Henn FA, Vollmayr B (2005). Stress models of depression: forming genetically vulnerable strains. Neurosci Biobehav Rev 29: 799-804.
Kelly JB, Masterton B (1977). Auditory sensitivity of the albino rat. J Comp Physiol Psychol 91: 930-936.

Krishnan V, Nestler EJ (2008). The molecular neurobiology of depression. Nature 455: 894-902.

Kukolja J, Schlapfer TE, Keysers C, Klingmuller D, Maier W, Fink GR et al (2008). Modeling a negative response bias in the human amygdala by noradrenergic-glucocorticoid interactions. J Neurosci 28: 12868-12876.

Lawson C, MacLeod C, Hammond G (2002). Interpretation revealed in the blink of an eye: depressive bias in the resolution of ambiguity. J Abnorm Psychol 111: 321-328.

Opris I, Bruce CJ (2005). Neural circuitry of judgment and decision mechanisms. Brain Res Brain Res Rev 48: 509-526.

Overmier JB, Seligman ME (1967). Effects of inescapable shock upon subsequent escape and avoidance responding. J Comp Physiol Psychol 63: 28-33.

Paton JJ, Belova MA, Morrison SE, Salzman CD (2006). The primate amygdala represents the positive and negative value of visual stimuli during learning. Nature 439: 865-870.

Pizzagalli DA, Iosifescu D, Hallett LA, Ratner KG, Fava M (2008). Reduced hedonic capacity in major depressive disorder: evidence from a probabilistic reward task. J Psychiatr Res 43: 76-87.

Rosen JB, Donley MP (2006). Animal studies of amygdala function in fear and uncertainty: relevance to human research. Biol Psychol 73: 49-60.

Seligman ME, Rosellini RA, Kozak MJ (1975). Learned helplessness in the rat: time course, immunization, and reversibility. J Comp Physiol Psychol 88: 542-547.

Shumake J, Poremba A, Edwards E, Gonzalez-Lima F (2000). Congenital helpless rats as a genetic model for cortex metabolism in depression. Neuroreport 11: 3793-3798.

Slattery DA, Markou A, Cryan JF (2007). Evaluation of reward processes in an animal model of depression. Psychopharmacology (Berl) 190: 555-568.

Sugrue LP, Corrado GS, Newsome WT (2005). Choosing the greater of two goods: neural currencies for valuation and decision making. Nat Rev Neurosci 6: 363-375.

Tsetsenis T, Ma XH, Lo IL, Beck SG, Gross C (2007). Suppression of conditioning to ambiguous cues by pharmacogenetic inhibition of the dentate gyrus. Nat Neurosci 10: 896-902.

Vollmayr B, Henn FA (2001). Learned helplessness in the rat: improvements in validity and reliability. Brain Res Brain Res Protoc 8: 1-7.

Wolfensberger SP, Veltman DJ, Hoogendijk WJ, Boomsma DI, de Geus EJ (2008). Amygdala responses to emotional faces in twins discordant or concordant for the risk for anxiety and depression. Neuroimage 41: 544-552.

Supplementary Information accompanies the paper on the Neuropsychopharmacology website (http://www.nature.com/npp) 